\title{
Protective effect of senegenin on splenectomy-induced postoperative cognitive dysfunction in elderly rats
}

\author{
LUYUAN YU ${ }^{1}$, LEI SUN ${ }^{2}$ and SULI CHEN ${ }^{3}$ \\ ${ }^{1}$ Department of Anesthesiology, Haidian Hospital, Haidian, Beijing 100080; ${ }^{2}$ Department of Anesthesiology, \\ Coal General Hospital, Chaoyang, Beijing 100028; ${ }^{3}$ Department of Anesthesiology, \\ Beijing Aerospace General Hospital, Fengtai, Beijing 100076, P.R. China
}

Received June 14, 2013; Accepted October 16, 2013

DOI: $10.3892 /$ etm.2014.1501

\begin{abstract}
Postoperative cognitive dysfunction (POCD) is common in elderly patients. Senegenin, an active component of extracts from Polygala tenuifolia root, a traditional Chinese medicine, has neuroprotective and neuroregenerative effects. However, the mechanism underlying the effects of senegenin against postoperative cognitive impairment in elderly individuals has yet to be elucidated. The aim of this study was to investigate the protective effects of senegenin on the cognitive functions of elderly rats with splenectomy-induced POCD. Results from a Morris water maze test suggested that splenectomy induced a transient cognitive deficiency in the elderly rats; however, when the rats were treated with senegenin, the cognitive impairment was notably attenuated. Further experiments showed that senegenin significantly inhibited the mRNA and protein expression of several key pro-inflammatory cytokines, specifically, tumor necrosis factor- $\alpha$ (TNF- $\alpha$ ), interleukin-1 $\beta$ (IL-1 $\beta$ ), IL-6 and IL-8, in the hippocampal tissues of elderly rats following splenectomy. In order to investigate the molecular mechanism involved, the expression and activity of the Toll-like receptor 4 (TLR4) signaling pathway was assessed. On day 1 postoperatively, it was observed that senegenin markedly suppressed the mRNA and protein expression of TLR4, myeloid differentiation factor 88 (MyD88) and TIR domain-containing adaptor-inducing interferon- $\beta$ (TRIF). Furthermore, the phosphorylation levels of nuclear factor $-\kappa \mathrm{B}(\mathrm{NF}-\kappa \mathrm{B})$ p 65 and inhibitor of $\mathrm{NF}-\kappa \mathrm{B}(\mathrm{I} \kappa \mathrm{B} \alpha)$ were also decreased following senegenin treatment on the first day subsequent to surgery. These results suggest that senegenin suppressed splenectomy-induced transient cognitive impairment in elderly rats, possibly by downregulating two signaling pathways involved in inflammation, TLR4/MyD88/NF-кB
\end{abstract}

Correspondence to: Professor Suli Chen, Department of Anesthesiology, Beijing Aerospace General Hospital, 7 Wanyuanbei Road, Fengtai, Beijing 100076, P.R. China

E-mail: anesthesiologycsl@163.com

Key words: postoperative cognitive dysfunction, senegenin, elderly, pro-inflammatory cytokines, signaling pathways and TLR4/TRIF/NF- $\kappa \mathrm{B}$, to further inhibit the expression of key pro-inflammatory cytokines, specifically, TNF- $\alpha$, IL- $1 \beta$, IL-6 and IL-8, and ultimately the neuroinflammation in the hippocampal tissues. In conclusion, the present study revealed that senegenin exhibited neuroprotective effects against splenectomy-induced transient cognitive impairment in elderly rats, which indicated that senegenin may be a promising agent for the treatment of POCD.

\section{Introduction}

Postoperative cognitive dysfunction (POCD), a decline in cognitive function following surgery, is characterized by an impairment of comprehension, concentration, and memory $(1,2)$. Although the molecular mechanism of POCD has yet to be elucidated, surgical trauma has been suggested to be a prominent risk factor for the development of POCD, possibly through the activation of signaling pathways involved in inflammation, such as the Toll-like receptor 4 (TLR4)/myeloid differentiation factor 88 (MyD88)/nuclear factor- $\kappa \mathrm{B}(\mathrm{NF}-\kappa \mathrm{B})$ and TLR4/TIR domain-containing adaptor inducing interferon $\beta$ (TRIF)/NF- $\kappa \mathrm{B}$ pathways (3). It has been demonstrated that the release of pro-inflammatory cytokines, such as tumor necrosis factor- $\alpha$ (TNF- $\alpha)$ and interleukin- $1 \beta$ (IL-1 $\beta$ ), increases following surgery $(4,5)$. These pro-inflammatory cytokines may trigger broad neuroinflammation in the brain (6). Thus, the effective inhibition of the activity of signaling pathways involved in inflammation and pro-inflammatory cytokine expression shows promise for the prevention and treatment for POCD.

Senegenin, also known as tenuigenin, is an active component of extracts from Polygala tenuifolia root, a traditional Chinese medicine, which has been shown to have neuroprotective and neuroregenerative effects, and is thus used to treat patients with insomnia, neurosis or dementia $(7,8)$. Recently, senegenin was demonstrated to attenuate hepatic ischemia-reperfusion-induced POCD by increasing hippocampal NR2B expression in rats (9); however, whether senegenin has a protective effect in splenectomy-induced cognitive dysfunction, as well as the molecular mechanism involved, has yet to be elucidated.

In the present study, to investigate the protective effects of senegenin on splenectomy-induced POCD, the cognitive 
functions of elderly rats following surgery with or without senegenin administration were studied. Following this, in order to investigate the molecular mechanism involved, the mRNA and protein expression levels of key pro-inflammatory cytokines, specifically, TNF- $\alpha$, IL-1 $\beta$, IL-6 and IL-8, were analyzed in the hippocampal tissues of elderly rats following splenectomy and senegenin administration. In addition, the

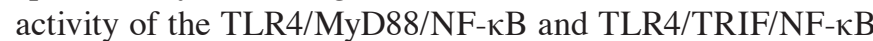
signaling pathways, which are crucial in neuroinflammation, were examined.

\section{Materials and methods}

Animals and groups. The animal experiments were performed in accordance with the Guidelines for the Care and Use of Laboratory Animals from the Ethics Committee of Beijing Handian District Hospital (Beijing, China). A total of 140 Sprague-Dawley male rats (25 months old, weighing 250-300 g) were purchased from the Shanghai Laboratory Animal Center of the Shanghai Institutes for Biological Sciences (Shanghai, China). The elderly rats were fed separately in conditions with a controlled temperature $\left(25-27^{\circ} \mathrm{C}\right)$ and illumination (12-h light/dark cycle) and were allowed free to standard rat chow and sterile water.

The 140 rats were randomly assigned to seven groups, each containing 20 rats. In the control group (Group C), the rats were not subjected to surgery. The remaining 120 rats were divided into surgery (Group S1, S3 and S5) and senegenin treatment (Group T1, T3 and T5) groups. All the rats were trained in the Morris water maze (MWM) for five days. Following training, the rats in Group $S$ underwent splenectomy under anesthesia without senegenin treatment, while the rats in Group $\mathrm{T}$ underwent splenectomy under anesthesia and were then treated with $60 \mathrm{mg} / \mathrm{kg}$ senegenin by gavage, once per day, continuously until day 5 after surgery. All rats were re-assessed using the MWM on days 1, 3 and 5 following surgery, respectively. This study was approved by the Ethics Committee of Beijing Aerospace General Hospital (Beijing, China).

MWM test. All the elderly rats were trained in the MWM with five trials/day for five consecutive days. A platform was placed in the center of the MWM. Each rat was placed on the platform for $30 \mathrm{sec}$ to ensure that the rat was aware of the existence of the platform. Following this, the rat was released into the water from a randomly assigned release point. The rat then had to find the platform and land on it within $60 \mathrm{sec}$. If the rat failed, it was picked up and placed on the platform for $30 \mathrm{sec}$. The rat was able to remain on the platform for $30 \mathrm{sec}$ between trials. Swimming distance, speed and latency to the platform were recorded by video tracking mounted on the ceiling. On days 1, 3 and 5 postoperatively, respectively, all the rats were re-assessed using the MWM test, in order to evaluate their partial memory and learning abilities.

RNA extraction and quantitative reverse transcription-polymerase chain reaction ( $q P C R)$. On days 1,3 and 5 postoperatively, total RNA was extracted using TRIzol ${ }^{\circledR}$ reagent (Invitrogen Life Technologies, Carlsbad, CA, USA) from the hippocampi of three rats randomly selected from each group. The RNA was then reverse transcribed into a cDNA template using a
PrimeScript ${ }^{\mathrm{TM}}$ reverse transcription (RT) reagent kit (Takara, Shiga, Japan). The cDNA was amplified using SYBR-Green qPCR Master mix (Invitrogen Life Technologies) for qPCR analysis using the ABI 7500 PCR system (Applied Biosystems, Foster City, CA, USA). The relative expression of target mRNA to glyceraldehyde 3-phosphate dehydrogenase (GAPDH) mRNA was calculated using crossing point $(\mathrm{Cp})$ values and scaled relative to control samples set at a value of 1 . The primer sequences used are shown as follows: TNF- $\alpha$ forward, 5'-CATGATCCGAGATGTGGAACTGGC-3' and reverse, 5'-CTGGCTCAGCCACTCCAGC-3'; IL-1 $\beta$ forward, 5'-CATGATCCGAGATGTGGAACTGGC-3' and reverse, 5'-CTGGCTCAGCCACTCCAGC-3'; IL-6 forward, 5'-ACTCACCTCTTCAGAACGAATTG-3' and reverse, 5'-CCATCTTTGGAAGGTTCAGGTTG-3'; IL-8 forward, 5'-TTTTGCCAAGGAGTGCTAAAGA-3' and reverse, 5'-AACCCTCTGCACCCAGTTTTC-3'; TLR4 reverse, 5'-ATGGCATGGCTTACACCACC-3' and reverse, 5'-GAGGCCAATTTTGTCTCCACA-3'; MyD88 forward, 5'-TCATGTTCTCCATACCCTTGGT-3' and reverse, 5'-AAACTGCGAGTGGGGTCAG-3'; TRIF forward, 5'-GCCAGCAACTTGGAAATCAGC-3' and reverse, 5'-GGGGTCGTCACAGAGCTTG-3'; GAPDH forward, 5'-GGAGCGAGATCCCTCCAAAAT-3' and reverse, 5'-GGCTGTTGTCATACTTCTCATGG-3'.

Immunohistochemical analysis in rat hippocampal tissues. All experimental rats in each group were sacrificed under anesthesia following the MWM test. The hippocampal tissues were rapidly separated, incubated with a peroxidase blocking solution, an avidin/biotin blocking solution and $10 \%$ fetal bovine serum (Invitrogen Life Technologies), prior to being cut into tissue sections. In order to examine the expression of TLR4, the hippocampal tissues sections were incubated with a TLR4-specific primary antibody, followed by incubation with an appropriate secondary antibody (goat anti-mouse secondary antibody; each from Santa Cruz Biotechnology, Inc., Santa Cruz, CA, USA).

Protein extraction and western blotting. On day 1 postoperatively, total protein was extracted using cold radio-immunoprecipitation assay (RIPA) lysis buffer from the hippocampi of three rats randomly selected from each group. Following this, the protein concentration was measured using a Bicinchoninic Acid protein assay kit (Pierce Biotechnology, Rockford, IL, USA). For the western blot analysis, $20 \mu$ g protein from each sample was separated using 5\% sodium dodecyl sulfate polyacrylamide gel electrophoresis (SDS-PAGE) and then transferred to a polyvinylidene difluoride (PVDF) membrane. The membranes were blocked in 5\% non-fat dried milk in phosphate-buffered saline (PBS) for $2 \mathrm{~h}$ and then incubated overnight with specific primary antibodies (mouse anti-TLR4 monoclonal antibody, mouse anti-MyD88 monoclonal antibody, rabbit anti-TRIF monoclonal antibody, rabbit anti-I $\kappa \mathrm{B} \alpha$ polyclonal antibody, mouse anti-p-p65 NF- $\kappa \mathrm{B}$ polyclonal antibody, mouse anti-TNF $\alpha$ polyclonal antibody, mouse anti-IL-1 $\beta$ polyclonal antibody, mouse anti-IL6 monoclonal antibody and rabbit anti-IL-8 monoclonal antibody; Santa Cruz Biotechnology, Inc.). GAPDH was used as a control. Following incubation with the appropriate secondary antibody 

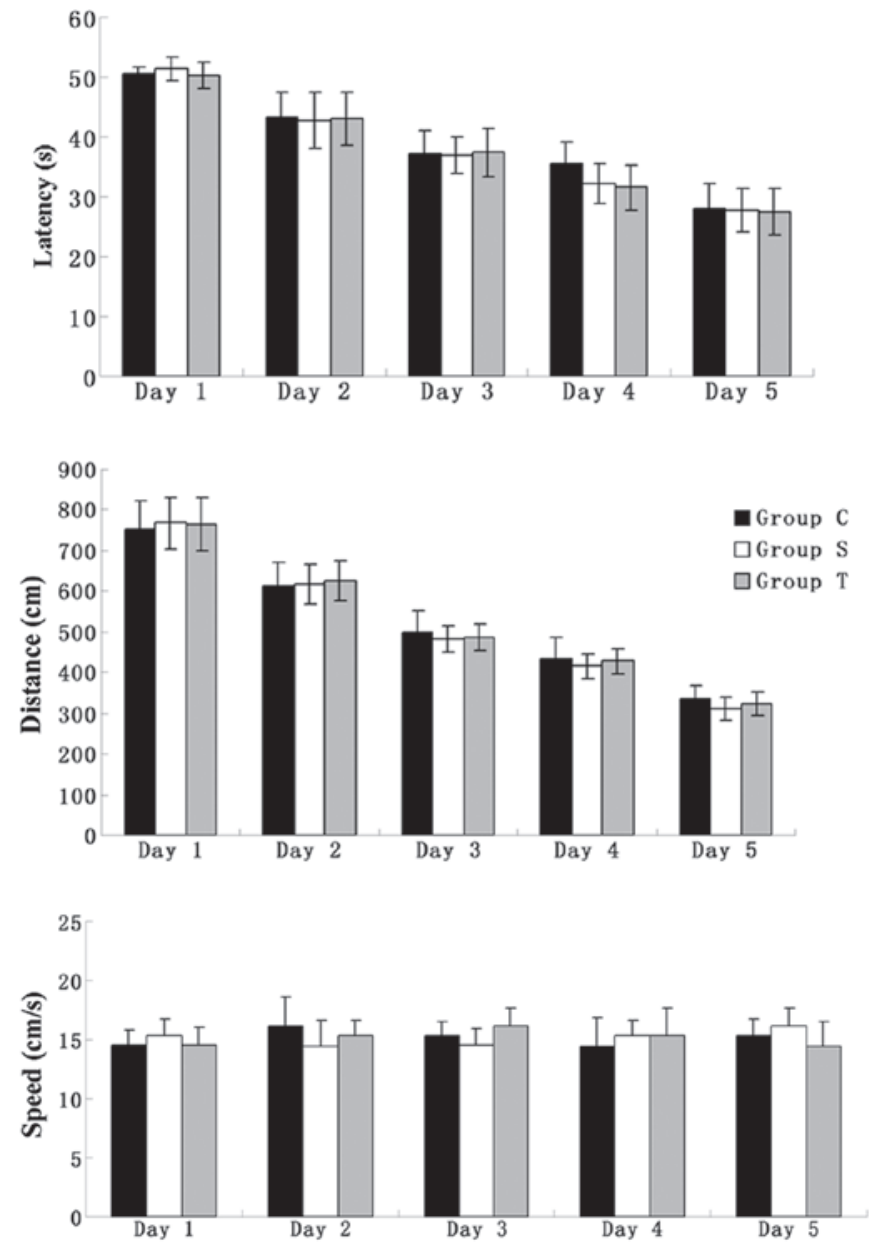

Figure 1. Swimming data (latency, swimming distance and swimming speed) of elderly rats for five consecutive training days in the Morris water maze prior to surgery. Group C, control group without surgery; Group S, surgery group without any treatment; Group T, surgery group with senegenin treatment. Results are presented as the mean \pm standard deviation.

(goat anti-mouse secondary antibody and mouse anti-rabbit secondary antibody; Santa Cruz Biotechnology, Inc.), immune complexes were assessed using peroxidase and an enhanced chemiluminescence system (Pierce ECL Western Blotting Substrate; Pierce Biotechnology).

Statistical analysis. All data are expressed as the mean \pm standard deviation. The statistical comparisons between groups were conducted using repeated measures analysis of variance (ANOVA) followed by a Least Significant Difference (LSD) test. All analyses were performed using SPSS 17.0 statistical software (SPSS, Inc., Chicago, IL, USA). A value of $\mathrm{P}<0.05$ was considered to indicate a statistically significant difference.

\section{Results}

Senegenin attenuates transient POCD induced by splenectomy in elderly rats. All elderly rats were trained to find the platform in the MWM for five days prior to surgery. The parameters of latency and distance were used to evaluate the spatial memory ability of the rats. During the five days of training, despite the swimming speed remaining unchanged, the other two parameters showed a tendency to decrease and,
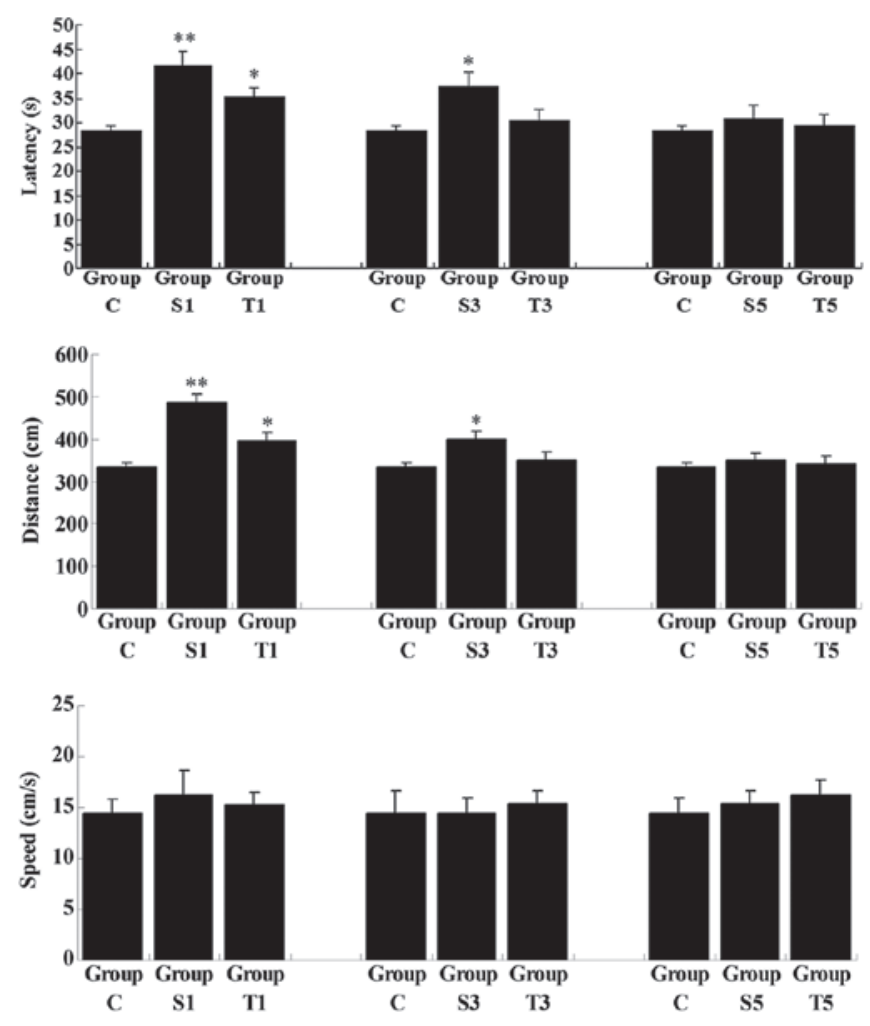

Figure 2. Swimming data (latency, swimming distance and swimming speed) of elderly rats following splenectomy in reversal learning testing. Group C, control group without surgery; Groups S1, S3 and S5, surgery group without any treatment on days 1, 3 and 5 postoperatively; Groups T1, T3 and T5, surgery group with senegenin treatment on days 1,3 and 5 postoperatively. Results are presented as the mean \pm standard deviation. ${ }^{*} \mathrm{P}<0.05$ and ${ }^{* *} \mathrm{P}<0.01$ compared with Group C.

in the fifth day of training, all the elderly rats were able to find the platform within $30 \mathrm{sec}$, indicating that their spatial memory had been consolidated (Fig. 1).

On day 1 following the splenectomy, despite the swimming speed remaining unchanged, the latency and swimming distance in the untreated group (Group S1) were significantly increased when compared with those in Group $\mathrm{C}(\mathrm{P}<0.01)$, revealing that the ability of the spatial memory was impaired shortly after splenectomy (Fig. 2). In the senegenin treatment group (Group T1), latency and swimming distance were also higher than those in Group C; however, the degree of increase was not as great as that in Group S1. On day 3 postoperatively, latency and swimming distance in the untreated group (Group S3), although reduced, remained higher than those in Group C $(\mathrm{P}<0.05)$, while these two parameters in Group T3 had returned to normal (compared with Group $\mathrm{C}, \mathrm{P}>0.05$ ). On day 5 following the splenectomy, latency and swimming distance in Groups $\mathrm{S}$ and $\mathrm{T}$ showed no significant differences from the results in Group $\mathrm{C}(\mathrm{P}>0.05)$, indicating that the spatial memory of the rats had returned to normal. These results suggested that splenectomy induced a transient cognitive deficit in elderly rats, and that senegenin had a potential therapeutic effect on this impairment.

Senegenin inhibits the mRNA and protein expression of pro-inflammatory cytokines in the hippocampi of elderly 

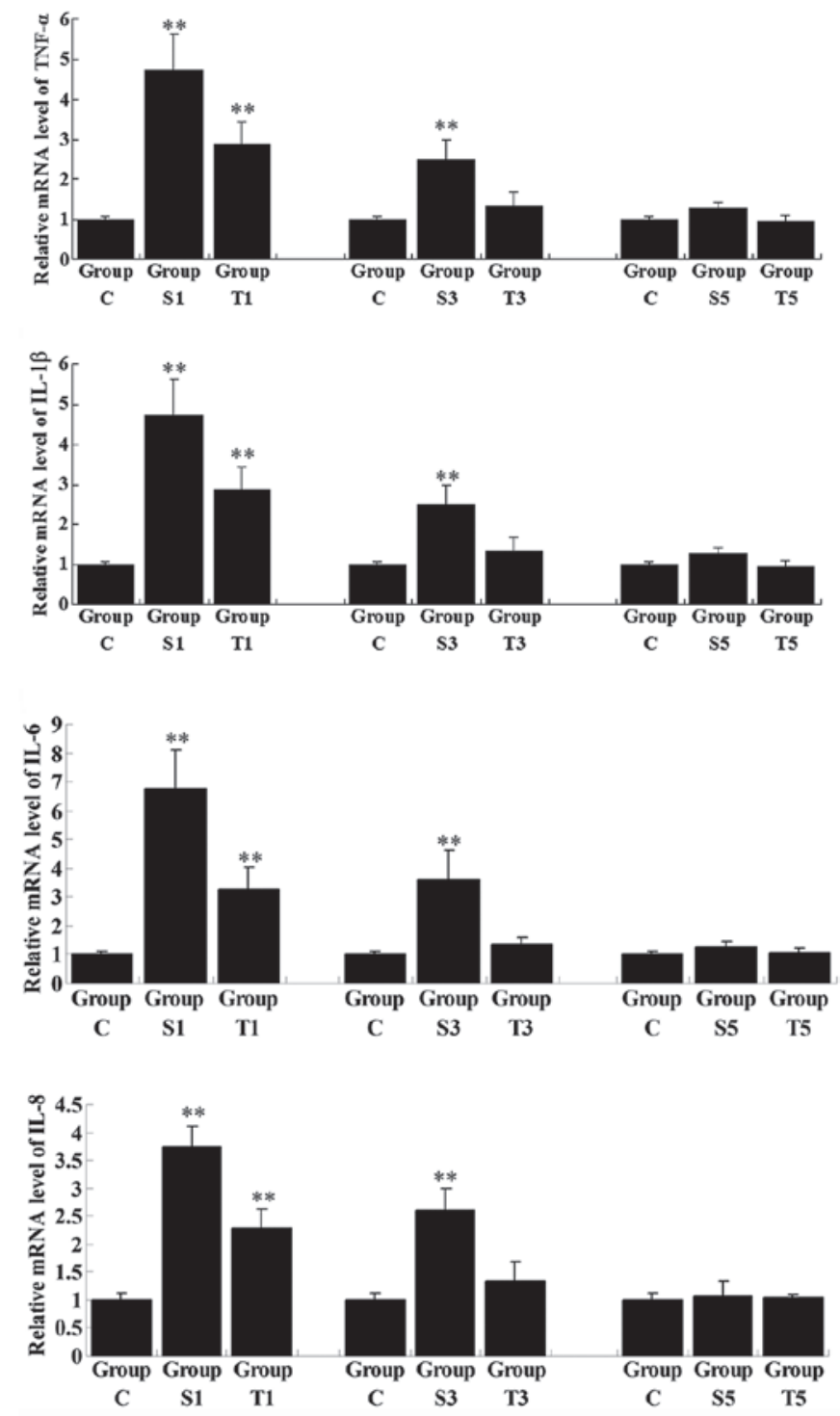

Figure 3. Quantitative reverse transcription-polymerase chain reaction (qPCR) assay for the relative mRNA expression of pro-inflammatory cytokines in rat hippocampal tissues. Group C, control group without surgery; Groups S1, S3 and S5, surgery group without any treatment on days 1, 3 and 5 postoperatively; Groups T1, T3 and T5, surgery group with senegenin treatment on days 1, 3 and 5 postoperatively. Results are presented as the mean \pm standard deviation. ${ }^{* *} \mathrm{P}<0.01$ compared with Group C. TNF- $\alpha$, tumor necrosis factor- $\alpha$; IL-1 $\beta$, interleukin- $1 \beta$.

rats following splenectomy. The mRNA and protein expression levels of a number of key pro-inflammatory cytokines, i.e., TNF- $\alpha$, IL- $1 \beta$, IL- 6 and IL-8, were determined in the hippocampi of elderly rats following surgery, with or without senegenin treatment. The mRNA expression levels of all these pro-inflammatory cytokines in Group S1 were significantly upregulated on day 1 postoperatively when compared with those in Group $\mathrm{C}(\mathrm{P}<0.01$; Fig. 3). However, following senegenin administration, the mRNA expression levels of the pro-inflammatory cytokines in Group T1 were lower than those in Group S1, although higher than those in Group C $(\mathrm{P}<0.01)$. The expression of the pro-inflammatory cytokines gradually decreased with time following the surgery. On day 3 post-surgery, the mRNA expression levels of the pro-inflammatory cytokines had returned to normal in Group T3, while
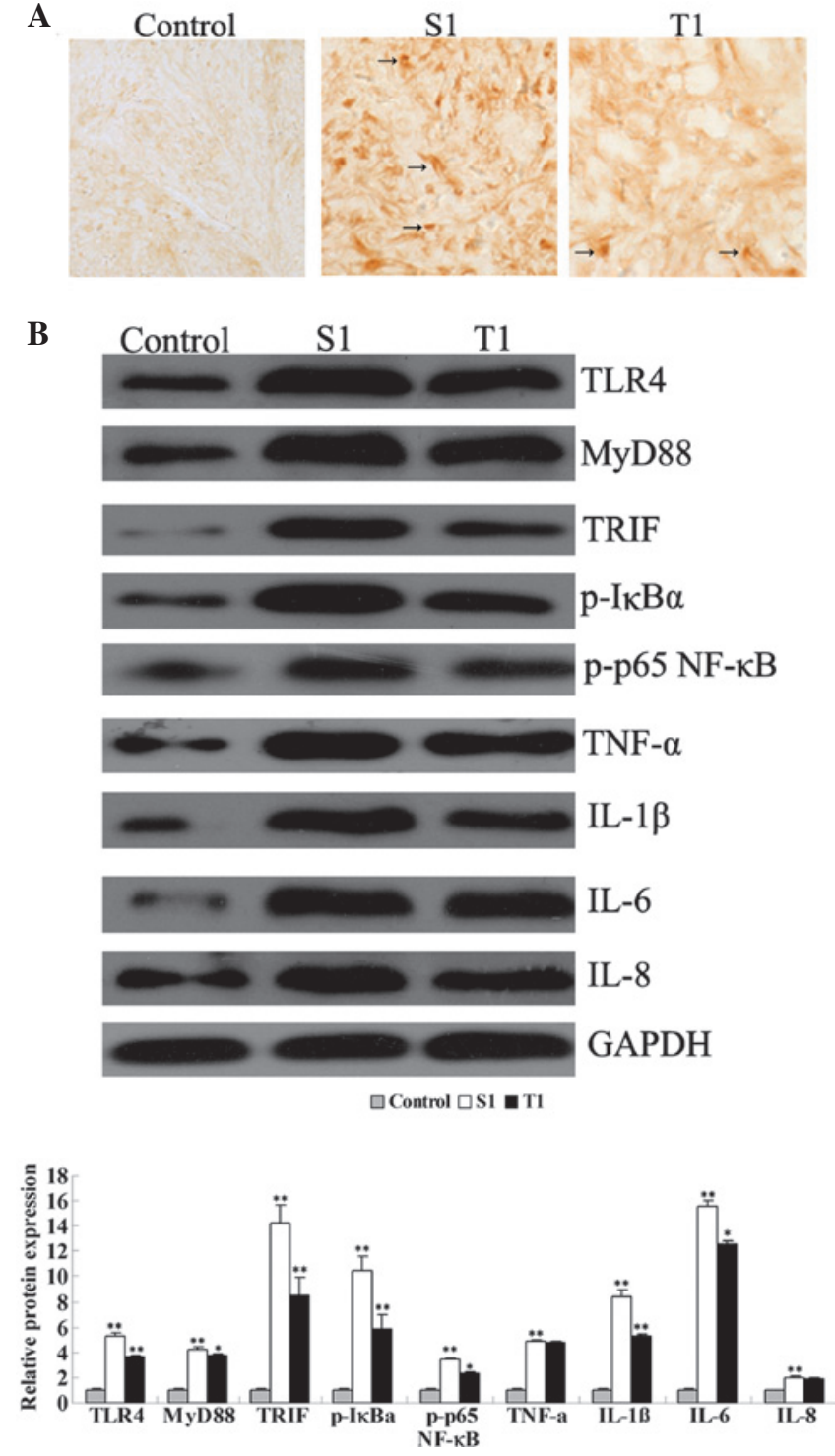

Figure 4. (A) Immunohistochemical analysis for Toll-like receptor 4 (TLR4) expression in rat hippocampal tissues (magnification, x200). (B) Western blot assay for the protein expression of key factors in the TLR4 signaling pathway and pro-inflammatory cytokines in rat hippocampal tissues. Group C, control group without surgery; Group S1, surgery group without any treatment on day 1 subsequent to surgery; Group T1, surgery group with senegenin treatment on day 1 postoperatively. ${ }^{*} \mathrm{P}<0.05$ and ${ }^{* * *} \mathrm{P}<0.01$ compared with Group $\mathrm{C}$. MyD88, myeloid differentiation factor 88; TRIF, TIR domain-containing adaptor inducing interferon- $\beta ; \mathrm{p}$-I $\mathrm{K} \mathrm{B} \alpha$, phosphorylated inhibitor of nuclear factor- $\kappa B(N F-\kappa B)$; p-p65 NF- $\kappa B$, phosphorylated p65 NF- $\kappa B$; TNF- $\alpha$, tumor necrosis factor- $\alpha$; IL-1 $\beta$, interleukin- $1 \beta$; GAPDH, glyceraldehyde 3-phosphate dehydrogenase.

the expression levels in Group S3 remained higher than those in Group $\mathrm{C}(\mathrm{P}<0.01)$. On day 5 postoperatively, the mRNA levels of the pro-inflammatory cytokines in Group S5 showed no significant differences from those in Group C $(\mathrm{P}>0.05)$. Western blot analyses were also performed to assess the protein expression levels of the pro-inflammatory cytokines on day 1 subsequent to surgery. The protein levels of IL-1 and IL-6 were consistent with the qPCR results $(\mathrm{P}<0.05)$, while the protein levels of TNF- $\alpha$ and IL- 8 were not $(\mathrm{P}>0.05)$; this may have been due to the post-transcriptional control of these two pro-inflammatory cytokines (Fig. 4). However, the results suggested that inflammation in the hippocampus following 

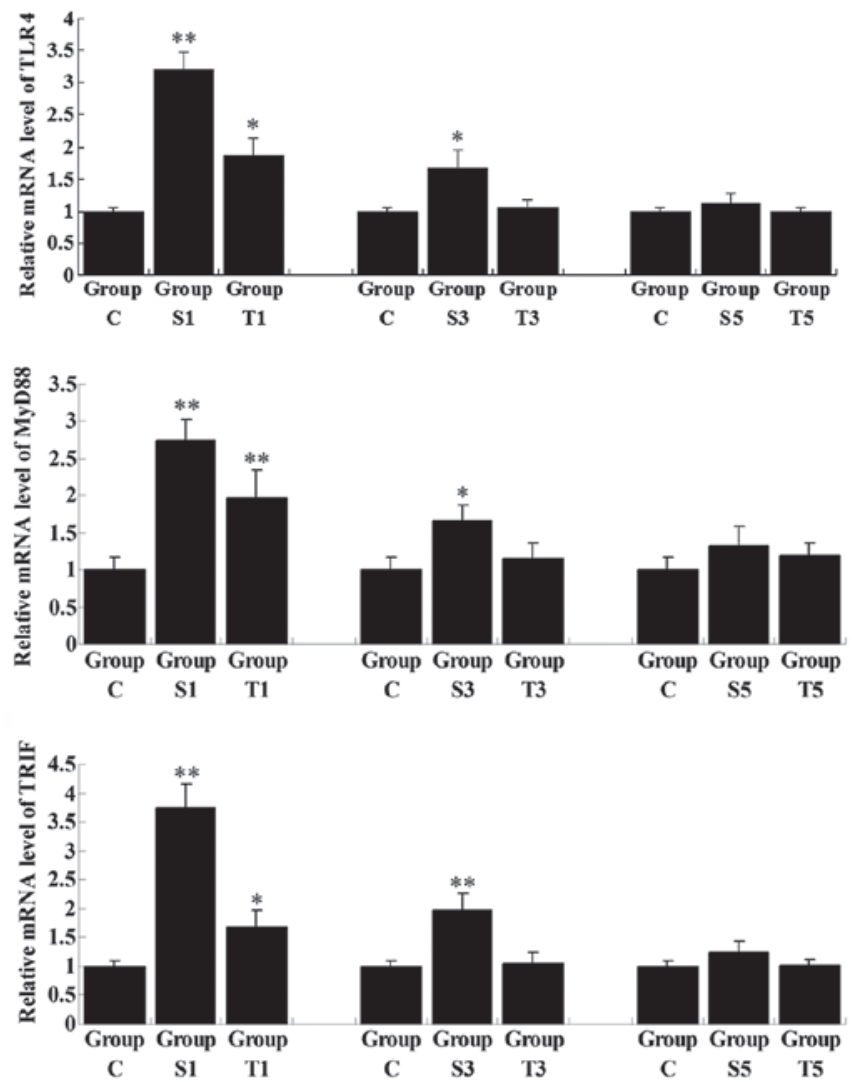

Figure 5. Quantitative reverse transcription-polymerase chain reaction assay for the relative mRNA expression of key factors in the Toll-like receptor 4 (TLR4) signaling pathway in rat hippocampal tissues. Group C, control group without surgery; Groups S1, S3 and S5, surgery group without any treatment on day 1, 3 and 5 postoperatively; Groups T1, T3 and T5, surgery group with senegenin treatment on days 1,3 and 5 postoperatively. Results are represented as the mean \pm standard deviation. ${ }^{*} \mathrm{P}<0.05$ and ${ }^{* *} \mathrm{P}<0.01 \mathrm{com}-$ pared with Group C. MyD88, myeloid differentiation factor 88; TRIF, TIR domain-containing adaptor inducing interferon- $\beta$.

surgery is crucial in the transient postoperative cognitive deficit and that the protective effect of senegenin against postoperative memory impairment in the hippocampi of elderly rats following splenectomy is partly due to its inhibitory effect on pro-inflammatory cytokine expression.

Senegenin inhibits signaling pathways involved in inflammation in the hippocampi of elderly rats. The activation of TLR4 is important in inflammation, and MyD88 and TRIF are key downstream components in the TLR4 signaling pathway, which is further able to activate $\mathrm{NF}-\kappa \mathrm{B}$, a family of transcription factors. NF- $\kappa \mathrm{B}$ regulates the transcription of a number of key inflammatory mediators, including TNF- $\alpha$, IL-1 $\beta$, IL-6 and IL-8 (10). To further investigate the regulatory role of senegenin in pro-inflammatory signaling pathways, the expression and activity of two signaling pathways involved in inflammation, i.e., TLR4/MyD88/NF- $\kappa \mathrm{B}$ and TLR4/TRIF/NF- $\kappa$ B, in the rat hippocampi in all the groups were examined. The mRNA expression levels of TLR4, MyD88 and TRIF in Group S1 were notably higher than those in Group C and in Group T1, in which group the rats were treated with senegenin shortly following surgery (Fig. 5). Consistent with the results of the MWM tests and qPCR for the pro-inflammatory cytokines, the expression levels of these key mediators in signaling pathways involved in inflammation were gradually decreased as time progressed following surgery. On day 3 subsequent to surgery, the expression levels of the cytokines had returned to normal in Group T3, although they remained higher in Group S3, when compared with those in Group C $(\mathrm{P}<0.05)$. On day 5 postoperatively, the expression levels in Group S5 showed no significant difference with those in Group C.

These results were further demonstrated by the immunohistochemical analysis and western blot assay. Group S1 showed a higher level of immunohistochemical staining for TLR4 than Group T1, indicating that, shortly following surgery, TLR4 was activated; however, senegenin was effectively able to inhibit the activation of TLR4 (Fig. 4). Furthermore, western blotting results showed that the protein expression levels of TLR4, MyD88 and TRIF were higher in Group S1 than those in Group T1 $(\mathrm{P}<0.05)$, consistent with the results of the qPCR. Moreover, the phosphorylation levels of $\mathrm{I} \kappa \mathrm{B} \alpha$ and p $65 \mathrm{NF}-\kappa \mathrm{B}$ were also higher in Group S1 than in Group T1, suggesting that on day 1 subsequent to surgery, $N F-\kappa B$ was activated in the hippocampus, while senegenin effectively suppressed its activation. All these results indicate that the effect of senegenin against surgery-induced neuroinflammation, as well as the transient cognitive impairment, may be attributed to its inhibitory roles in the TLR4/MyD88/NF- $\kappa \mathrm{B}$ and TLR4/TRIF/NF- $\kappa \mathrm{B}$ signaling pathways, which are involved in inflammation.

\section{Discussion}

POCD, a decline in cognitive function following surgery, is commonly observed in elderly patients (11). It has been demonstrated that extracts from Polygala tenuifolia root are able to promote memory in healthy individuals and enhance cognitive functions in elderly patients $(12,13)$. Senegenin is the active component of the Polygala tenuifolia root extracts, and has been shown to attenuate hepatic ischemia-reperfusion-induced cognitive deficiency by increasing hippocampal NR2B expression in rats (9). However, the molecular mechanism underlying the effect of senegenin on POCD in elderly individuals has not been fully elucidated. In the present study, a splenectomy model in rats was used to perform an investigation into POCD. The data in this study showed that splenectomy induced postoperative cognitive impairment, and that senegenin exhibited a therapeutic effect on this cognitive deficiency.

It has been reported that POCD may be attributed to a number of potential factors, including a noninfectious neuroinflammatory response, while surgery is able to induce neuroinflammation $(4,14)$. Accordingly, the expression levels of pro-inflammatory cytokines were further investigated to evaluate the neuroinflammatory response induced by splenectomy in elderly rats. The results showed increasing expression levels of the pro-inflammatory cytokines TNF- $\alpha$, IL-1 $\beta$, IL-6 and IL-8 in the hippocampi of elderly rats following splenectomy. This was consistent with other studies, which demonstrated that, following peripheral surgery, inflammatory activation was present in the hippocampus and that the expression levels of pro-inflammatory cytokines were upregulated in the hippocampus $(15,16)$.

TLR4, a pattern recognition receptor, participates in the inflammatory response by producing numerous pro-inflamma- 
tory factors via MyD88-dependent and MyD88-independent pathways, which are further able to activate $\mathrm{NF}-\kappa \mathrm{B}(17,18)$. $\mathrm{NF}-\kappa \mathrm{Bs}$ are a family of transcription factors that regulate the mRNA transcription of multiple pro-inflammatory cytokines, including TNF- $\alpha$, IL-1 $\beta$, IL- 6 and IL-8 $(19,20)$. Thus, the $\mathrm{NF}-\kappa \mathrm{B}$ signaling pathway is pivotal in inflammation. Under normal conditions, $\mathrm{NF}-\kappa \mathrm{B}$ interacts with $\mathrm{I} \kappa \mathrm{B} \alpha$ and is located in the cytoplasm. As I $\kappa \mathrm{B} \alpha$ is phosphorylated, it becomes ubiquitinated and is eventually degraded by the $26 \mathrm{~S}$ proteasome (21). As a result of this, the nuclear localization signal of $\mathrm{NF}-\kappa \mathrm{B}$ becomes unmasked. NF- $\kappa \mathrm{B}$ may then enter into the nucleus and promote the transcription of its targets, including TNF- $\alpha$, IL-1 $\beta$, IL-6 and IL-8 (22). In the present study, the increased mRNA and protein expression levels of TLR4, MyD88 and TRIF in the hippocampus following surgery were effectively suppressed by senegenin. Moreover, the phosphorylation levels of $\mathrm{I} \kappa \mathrm{B} \alpha$ and p $65 \mathrm{NF}-\kappa \mathrm{B}$ were significantly upregulated in the rat hippocampus on the first day following splenectomy; however, when the rats were treated with senegenin, the phosphorylation levels of $\mathrm{I} \kappa \mathrm{B} \alpha$ and p $65 \mathrm{NF}-\kappa \mathrm{B}$ were markedly lower than those in Group S1, indicating that senegenin further suppressed the activation of $N F-\kappa B$ by inhibiting the phosphorylation of $\mathrm{I} \kappa \mathrm{B} \alpha$ and then the translocation of $\mathrm{NF}-\kappa \mathrm{B}$ into the nucleus. In combination, these results suggest that the effect of senegenin against neuroinflammation may have been mediated through the inhibition of the TLR4/MyD88/NF- $\mathrm{B}$ and TLR4/TRIF/NF- $\kappa \mathrm{B}$ signaling pathways, which are involved in inflammation.

In conclusion, the present study revealed the neuroprotective effect of senegenin in splenectomy-induced cognitive impairment in elderly rats, mediated through inhibition of the inflammation-related TLR4/MyD88/NF- $\kappa \mathrm{B}$ and TLR4/TRIF/NF- $\kappa \mathrm{B}$ signaling pathways, the downregulation of pro-inflammatory cytokine expression and, ultimately, the suppression of neuroinflammation in the brain. The present study indicated that senegenin exhibited promising preventive and therapeutic effects for POCD in the elderly.

\section{References}

1. Coburn M, Fahlenkamp A, Zoremba N and Schaelte G: Postoperative cognitive dysfunction: Incidence and prophylaxis. Anaesthesist 59: 177-185, 2010.

2. Deiner S and Silverstein JH: Postoperative delirium and cognitive dysfunction. Br J Anaesth 103 (Suppl 1): i41-i46, 2009.

3. Hu Z, Ou Y, Duan K and Jiang X: Inflammation: a bridge between postoperative cognitive dysfunction and Alzheimer's disease. Med Hypotheses 74: 722-724, 2010.

4. Rosczyk HA,Sparkman NL and Johnson RW: Neuroinflammation and cognitive function in aged mice following minor surgery. Exp Gerontol 43: 840-846, 2008.
5. Terrando N, Monaco C, Ma D, Foxwell BM, Feldmann M and Maze M: Tumor necrosis factor-alpha triggers a cytokine cascade yielding postoperative cognitive decline. Proc Natl Acad Sci USA 107: 20518-20522, 2010.

6. Teeling JL and Perry VH: Systemic infection and inflammation in acute CNS injury and chronic neurodegeneration: underlying mechanisms. Neuroscience 158: 1062-1073, 2009.

7. Park CH, Choi SH, Koo JW, et al: Novel cognitive improving and neuroprotective activities of Polygala tenuifolia Willdenow extract, BT-11. J Neurosci Res 70: 484-492, 2002.

8. Ikeya Y, Takeda S, Tunakawa M, et al: Cognitive improving and cerebral protective effects of acylated oligosaccharides in Polygala tenuifolia. Biol Pharm Bull 27: 1081-1085, 2004.

9. Xie W, Yang Y, Gu X, et al: Senegenin attenuates hepatic ischemia-reperfusion induced cognitive dysfunction by increasing hippocampal NR2B expression in rats. PLoS One 7: e45575, 2012.

10. DiDonato JA, Mercurio F and Karin M: NF- $\kappa B$ and the link between inflammation and cancer. Immunol Rev 246: 379-400, 2012.

11. Hartholt KA, van der Cammen TJ and Klimek M: Postoperative cognitive dysfunction in geriatric patients. Z Gerontol Geriatr 45: 411-416, 2012.

12. Shin KY, Lee JY, Won BY, et al: BT-11 is effective for enhancing cognitive functions in the elderly humans. Neurosci Lett 465 : 157-159, 2009.

13. Lee JY, Kim KY, Shin KY, Won BY, Jung HY and Suh YH: Effects of BT-11 on memory in healthy humans. Neurosci Lett 454: 111-114, 2009.

14. Haseneder R, Kochs E and Jungwirth B: Postoperative cognitive dysfunction. Possible neuronal mechanisms and practical consequences for clinical routine. Anaesthesist 61: 437-443, 2012 (In German).

15. Hua F, Ma J, Ha T, et al: Activation of Toll-like receptor 4 signaling contributes to hippocampal neuronal death following global cerebral ischemia/reperfusion. J Neuroimmunol 190: 101-111, 2007.

16. Barrientos RM, Hein AM, Frank MG, Watkins LR and Maier SF: Intracisternal interleukin-1 receptor antagonist prevents postoperative cognitive decline and neuroinflammatory response in aged rats. J Neurosci 32: 14641-14648, 2012.

17. Crack PJ and Bray PJ: Toll-like receptors in the brain and their potential roles in neuropathology. Immunol Cell Biol 85: 476-480, 2007.

18. Okun E, Griffioen KJ, Lathia JD, Tang SC, Mattson MP and Arumugam TV: Toll-like receptors in neurodegeneration. Brain Res Rev 59: 278-292, 2009.

19. Sohn KH, Jo MJ, Cho WJ, et al: Bojesodok-eum, a herbal prescription, ameliorates acute inflammation in association with the inhibition of NF- $\mathrm{kB}$-mediated nitric oxide and proinflammatory cytokine production. Evid Based Complement Alternat Med 2012: 457370, 2012.

20. Cortez M, Carmo LS, Rogero MM, Borelli P and Fock RA: A high-fat diet increases IL-1, IL- 6 , and TNF- $\alpha$ production by increasing NF- $\kappa \mathrm{B}$ and attenuating PPAR- $\gamma$ expression in bone marrow mesenchymal stem cells. Inflammation 36: 379-386, 2013.

21. Iwai K: Diverse ubiquitin signaling in NF- $\kappa$ B activation. Trends Cell Biol 22: 355-364, 2012.

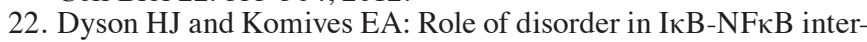
action. IUBMB Life 64: 499-505, 2012. 